\title{
MIRR - Model-based Instrument for River Restoration. Entwicklung eines strategischen Instru- ments zur integrativen Bewertung ökologischer Restaurationsmaßnahmen an Fließgewässern
}

MIRR - Model-based Instrument for River Restoration. Developing a strategic instrument for the integrated assessment of ecological stream restoration measures

\section{von S. Schmutz, A. Melcher, S. Muhar, A. Zitek, M. Poppe, C. Trautwein und M. Jungwirth}

\section{Kurzfassung/Summary}

Die Fließgewässer Österreichs sind vielfältigen anthropogenen Beeinträchtigungen ausgesetzt. Während die Gewässergüte nicht mehr zu den prioritären Problemen zählt, weisen die meisten Gewässer hydromorphologische Veränderungen auf. Zur Wiederherstellung des „guten ökologischen Zustandes“ bzw. des "guten ökologischen Potentials“ im Sinne der Wasserrahmenrichtlinie sind daher für diese Gewässer geeignete Sanierungsmaßnahmen festzulegen.

Aus einer Vielzahl fallspezifischer Einzeluntersuchungen ist bekannt, dass Fische sehr gut hydromorphologische Belastungen anzeigen. Bislang wurde jedoch in Österreich noch kein Versuch unternommen, anhand eines größeren Datensatzes die Wirkungszusammenhänge zwischen hydromorphologischen Belastungen und Fischen systematisch zu untersuchen und die daraus abgeleiteten Erkenntnisse zur Formulierung von Sanierungsstrategien und Restaurationsmaßnahmen heranzuziehen.

Das Bundesministerium für Land- und Forstwirtschaft, Umwelt und Wasserwirtschaft (Sektion Wasser, Ref. VII 1 b - Gewässerökologie und VII-5 - Schutzwasserwirtschaft) sowie die Niederösterreichische Landesregierung (Gruppe Wasser) beauftragten daher das Institut für Hydrobiologie und Gewässermanagement der Universität für Bodenkultur Wien mit der Erstellung der MIRR-Studie zur Entwicklung eines strategischen Instruments zur Restauration von Fließgewässern (Model-based In-
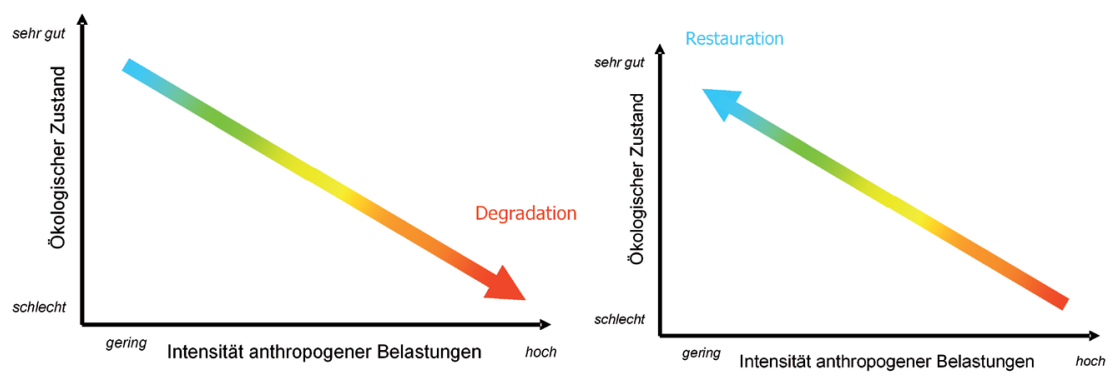

Abb. 1: Konzeptueller Ansatz des MIRR Projekts, dabei werden Degradationsprozesse stellvertretend für Restaurationsprozesse herangezogen

strument for River Restoraion) (Schmutz et al. 2007, http://mirr. boku.ac.at).

Da bislang erst wenige Restaurationsmaßnahmen in Österreich umgesetzt wurden und daher für statistische Analysen noch nicht ausreichend Datenmaterial zur Verfügung stand, wurde das Instrument anhand eines Analogieschlusses entwickelt. Die zugrunde liegende Hypothese ist, dass der ökologische Zustand der Gewässer sich bei Restauration in ähnlicher Weise verbessert, wie er sich durch die Belastungen verschlechterte. Das heißt, die Wegnahme der Belastung führt im Sinne einer Umkehrung des Degradationsprozesses über vergleichbare Mechanismen wieder zum unbelasteten Zustand (Abbildung 1).

Dieser Ansatz besitzt natürlich eingeschränkte Gültigkeit, da der Restaurationsprozess aufgrund mehrerer Ursachen anders als der Degradationsprozess verlaufen kann. Trotzdem entspricht dieser Ansatz dem belastungsorientiertem Konzept der Wasserrahmenrichtlinie und stellt aus unserer Sicht unter den gegebenen Rahmenbedingungen den einzigen möglichen Weg einer adäquaten methodischen Herangehensweise dar.

In 2000 the most modern water legislation in the world was launched by the European Union, the Water Framework Directive (WFD). One of its key objectives is to achieve the "good ecological status" of running waters by 2015. Therefore, in Austria currently a strategic instrument to identify hydro-morphological restoration measures for running waters was developed (MIRR-Model-based Instrument for River Restoration). As fish are known to be sensitive to hydro-morphological pressures, in particular continuum interruptions, the WFD considers fish as indicators for the ecological status of rivers. For analyzing the present status of the Austrian fish fauna with regard to typical hydro-morphological pressures, a database containing information on more than 700 fishing sites in Lower Austria was developed. Relevant criteria characterizing anthropogenic pressures such as hydrological alterations (water abstraction), impoundment, land use, channelisation, continuum interruptions (lateral, longitudinal) and water quality changes were selected based 
on an extensive literature search. Pressure data were collated using existing information and GIS tools. Multivariate analyses of the different types of pressures on different spatial and temporal scales allow for a quantification of relationships between pressures and fish, as well

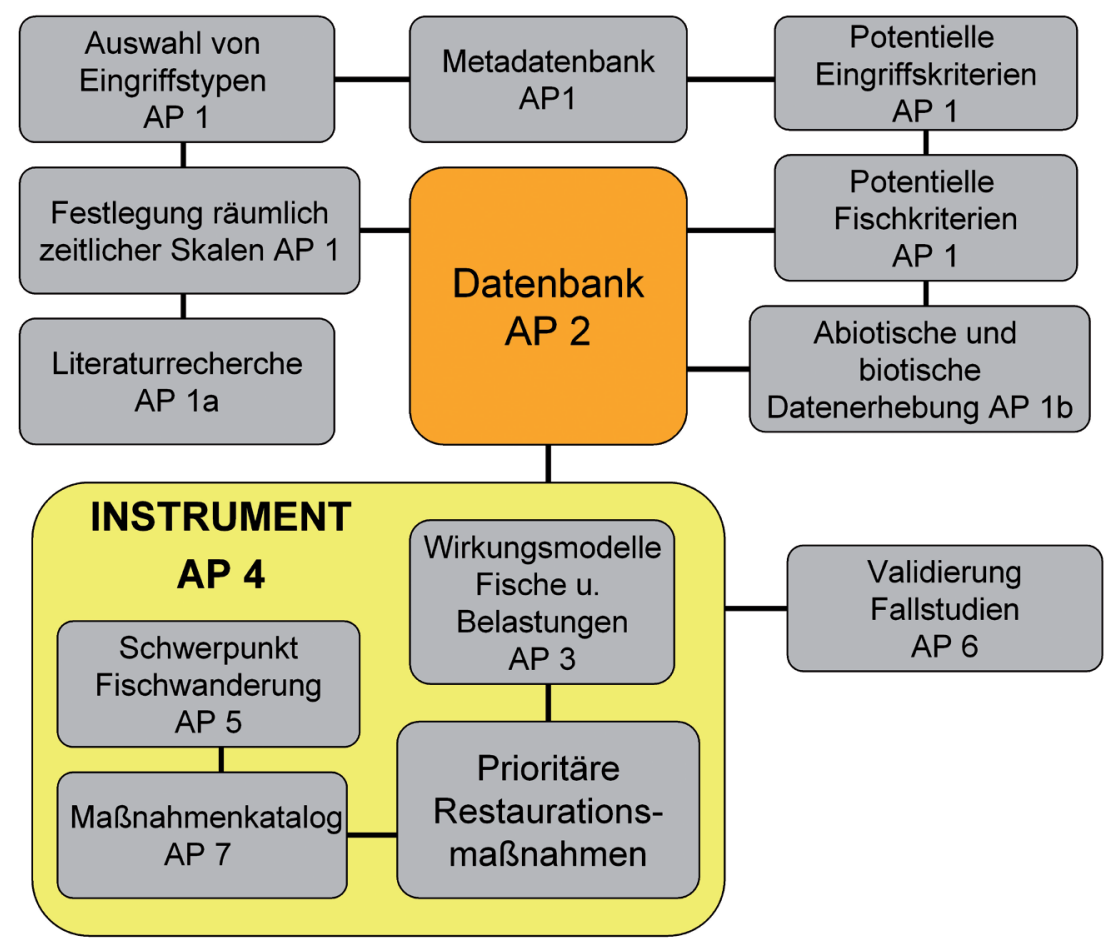

Abb. 2: Schematische Darstellung der MIRR Projektstruktur

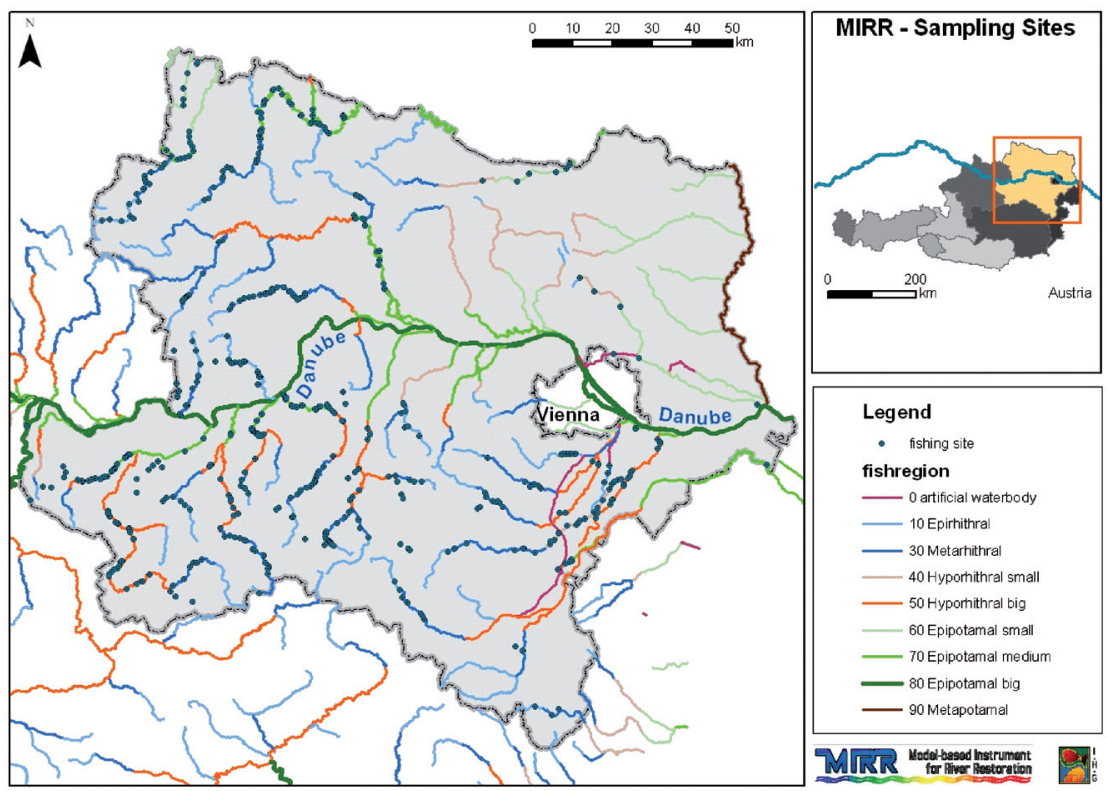

Abb. 3: Lage der Befischungspunkte des MIRR Projekts in Niederösterreich

\section{Projektstruktur und Untersuchungsgebiet}

Das MIRR Projekt gliedert sich, wie in Abbildung 2 dargestellt, in insgesamt 8 Arbeitspakete (AP). Zu Beginn des Projektes wurde im AP la anhand einer Literaturrecherche und unter Mithilfe von Expertenwissen eine umfangreiche Liste möglicher Belastungskriterien $\mathrm{zu}$ den Belastungstypen Regulierung, Kontinuumsunterbrechungen, Landnutzung, Stau, Restwasser, Schwall und sonstige erstellt. Der Bericht „Potential Pressure Criteria for Modelling Fish/Pressure Relationships in Running Waters - A Literature Review“ von A. Zitek wurde im Februar 2006 fertig gestellt und ist seitdem auch über die Internetseite des Lebensministeriums beziehbar: (wasser.lebensministerium.at/filemanager/download/14534/).

Da Belastungsdaten in Österreich nicht flächendeckend und nicht in konsistenter Form vorlagen, musste die Datenrecherche (AP 1b) auf Niederösterreich beschränkt werden (Abbildung 3). Für dieses Bundesland lagen die meisten Daten vor. Die Erfassung von Belastungsdaten für jene Gewässerabschnitte, an denen auch Befischungsdaten vorlagen, nahm den größten Teil der Arbeiten dieses Projekts ein. Unter anderem mussten fehlende Daten ergänzt oder im Freiland neu erhoben werden.

Insgesamt wurden in der MIRR Datenbank (AP 2) Fischbestände von 938 Beprobungen in 715 Probestellen erfasst. Für ca. 400 Stellen ließen sich auch weitgehend vollständige Belastungsdaten hinsichtlich Regulierung, Stau, Landnutzung, Kontinuum und Restwasser recherchieren. Zusätzlich wurden weitere Umweltparameter, wie Seehöhe oder Gefälle, zur Charakterisierung der Probenstellen erfasst.

Anhand recherchierter Daten 
wurden im 3. und 4. AP Wirkungsbeziehungen zwischen den Belastungen und der Reaktion der Fische analysiert und ein Instrument zur Identifizierung prioritärer Restaurationsmaßnahmen in beeinträchtigten Fließgewässern entwickelt.

Aufgrund der zentralen Bedeutung des Kontinuums für Fische und der vorgegebenen Zielsetzungen des Projektes wurde die Problematik der Sanierung von Migrationshindernissen in einem eigenen AP 5, im so genannten „Kontinuumsschwerpunkt" behandelt und zwecks Übersichtlichkeit in einem separaten Bericht veröffentlicht (Zitek et al. 2007 http://mirr.boku.ac.at).

In einer Fallstudie an der Traisen (AP 6) wurde das entwickelte Instrument getestet und mit anderen Methoden verglichen (Hohensinner et al. 2008).

Der Schwerpunkt der in AP 7 enthaltenen Maßnahmenbeschreibung liegt auf der Restaurierung gewässermorphologischer und hydrologischer Verhältnisse. Die Maßnahmen reichen von kleinräumigen Strukturierungen des Gewässerbettes bis hin zu vernetzenden Maßnahmen im Auenniveau sowie Maßnahmen zur Verbesserung gestörter hydrologischer Verhältnisse und betreffen damit unterschiedliche räumliche Ebenen.

AP 8 umfasst zum einen die gesamte interne Projektkoordination (insgesamt haben mehr als 30 KollegInnen des IHG mitgearbeitet), aber auch den fachlichen Austausch mit externeren ExpertInnen. Diese wurden in Form von 3 ExpertInnenworkshops in das Projekt eingebunden. TeilnehmerInnen der Workshops waren FachkollegInnen von Universitäten, Ministerien, Bundesämtern, Landesregierungen, Planungsbüros sowie Interessensvertretungen (E-Wirtschaft, Fischerei und Umweltverbände).

\section{Methodik}

Zur Beurteilung des fischökologischen Zustandes wurden Fischindizes herangezogen, die sich aus mehreren fischökologischen Kriterien, so genannten „Metrik“ zusammensetzten (Multimetrisches Verfahren). Die Metrik ergeben sich aus den ähnlichen Ansprüchen von Artengruppen (z.B. Anzahl strömungsliebender Arten), so genannten ökologischen Gilden. Zu Beginn wurde der Datensatz in geringer und stärker belastete Probenstellen untergliedert. Für jeden potentiellen Metrik wurde mit den geringer belasteten Probenstellen ein Regressionsmodell anhand von Umweltvariablen (Seehöhe, Gefälle, Distanz zur Quelle, etc.) entwickelt, um die natürliche Variabilität von Fischlebensgemeinschaften zu erfassen. Mittels dieser Regressionsmodelle ließ sich somit für jede stärker belastete Probenstelle ein „Referenzwert" für die Situation bei geringerer Belastung ermitteln und die Abweichung vom tatsächlichen Wert (Residuen) als Maß für den Beeinträchtigungsgrad berechnen. Reaktive Metrik wurden anhand des Vergleichs zwischen geringerer und stärkerer Belastung selektiert. Die jeweils 5 reaktivsten und nicht redundanten Metrik je Belastungstyp wurden zu belastungsspezifischen Fischindizes getrennt für Rhithral und Potamal zusammengefasst. Dies ergab für die 5 Belastungstypen 10 belastungsspezifische Indizes. Zusätzlich wurden generelle Indizes (ohne Unterscheidung des Belastungstyps) zu Vergleichszwecken entwickelt. Unter Zuhilfenahme einer Diskriminanzanlyse kann basierend auf den 10 belastungsspezifischen Indizes eine Vorhersage des zu erwartenden Belastungstyps erfolgen. Für die weiteren Analysen wurde ein Kombinierter-Fischindex aus den jeweils reaktivsten Indizes je Fischregion ermittelt. Mittels
Kombinierten-Fischindex (kurz Fischindex) und Regressionsbäumen wurden Effekte kombinierter Belastungen im Detail analysiert.

\section{Ergebnisse und Diskussion}

Zielsetzung des MIRR-Projektes war die Entwicklung eines Instruments für Sanierungsmaßnahmen. Dafür ist die Kenntnis der Wirkungszusammenhänge zwischen bestimmten Belastungen und den Fischen Voraussetzung. Aufgrund der Voranalysen zeigte es sich, dass Fische belastungsspezifisch reagieren. Daher wurden belastungsspezifische Indizes entwickelt.

\subsection{Fischindizes}

Die Ergebnisse zeigten, dass für die belastungsspezifischen Indizes nicht nur herkömmliche Metrik Verwendung fanden, sondern auch neue Metrikvarianten geeignet waren, Belastungen anzuzeigen. Die Hälfte der verwendeten Metrik basierte auf Biomassewerten. Ein Drittel war auf Flusslängen bezogen, ein Hinweis, dass die Verringerung des Lebensraumes, bezogen auf die Lauflänge, einen signifikanten Effekt bei Fischen zeigt. Bei zwei Drittel der Metrik wurden nur Teile der Population hinsichtlich der Längenverteilung verwendet $(<15 \mathrm{~cm},>20 \mathrm{~cm}$ Fischlänge). Bei bestimmten Belastungstypen reagieren größere Fische bestimmter Metrik stärker als kleinere, bei anderen ist dies wiederum umgekehrt.

Neben den belastungsspezifischen Indizes wurden auch Gesamt-Indizes (jeweils für Rhithral und Potamal) entwickelt, um diese mit den belastungsspezifischen Indizes vergleichen zu können. Die Gesamtindizes zeigten durchwegs eine geringere Fähigkeit, Belastungen zu erkennen. Dies war nur anhand der belastungsspezifischen Indizes möglich.

Ziel des MIRR-Projektes war nicht die Entwicklung einer fisch- 


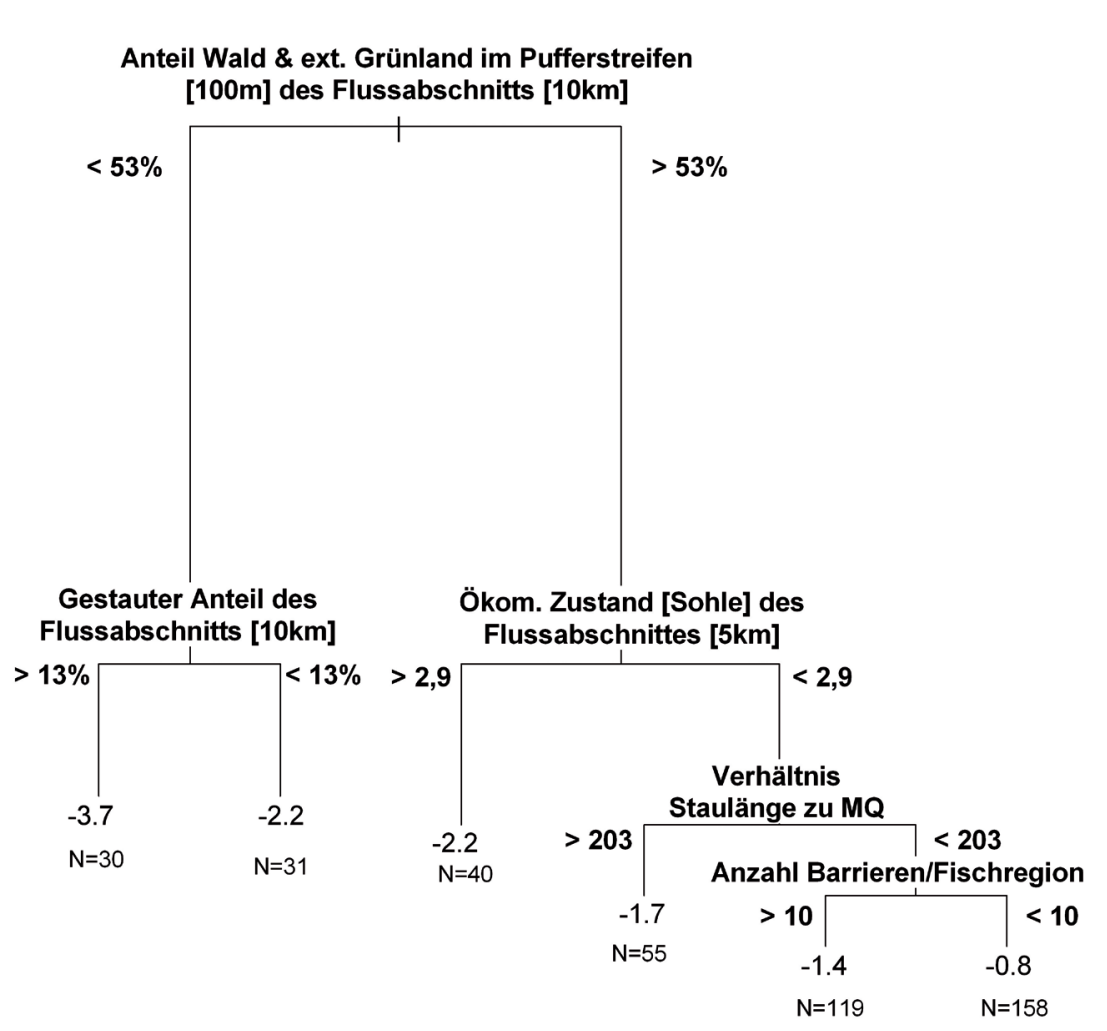

Abb. 4: Regressionsbaum kombinierter Belastungen, Grenzwerte für Belastungskriterien für die Aufzweigung des Baumes (links und rechts der Aufzweigung), durchschnittlicher Fischindex pro Ast

ökologischen Bewertungsmethode für die Beurteilung des ökologischen Zustandes gemäß Wasserrahmenrichtlinie, da es hierfür bereits eine nationale Methode gibt (Haunschmid et al. 2006). Freilich ist anzuregen, bei der Überarbeitung bzw. Weiterentwicklung der nationalen Methode die Erkenntnisse des MIRR-Projektes hinsichtlich belastungsspezifischer Indizes einfließen zu lassen.

\subsection{Mehrfach- und Einzelbelastungen}

Die Ergebnisse der Modellierungen zeigen sowohl bei der belastungsspezifischen Analyse, als auch bei der Beurteilung kombinierter Belastungen deutliche Unterschiede in der Reaktion der Fische auf Belastungen.

Am stärksten reagieren Fische auf Einzelbelastungen hinsichtlich Landnutzung und Kontinuum im Potamal. Insgesamt reagieren $\mathrm{Fi}$ - sche im Potamal wesentlich stärker auf Belastungen als im Rhithral.

Für die Beurteilung des fischökologischen Zustands einer Probenstelle wurde letztlich der Minimalwert aller belastungsspezifischen Indizes herangezogen. Dieser so genannte KombinierteIndex wurde für die Modellierung mittels Regressionsbaum-Analysen herangezogen. Sowohl die belastungsspezifischen Indizes als auch der Kombinierte-Index wurden für die Entwicklung des Instruments verwendet.

Anhand der belastungsspezifischen Indizes selbst lässt sich mittels Diskriminanzanalyse der maßgebliche Belastungstyp feststellen. Mit Hilfe von Diskriminanzanalysen können in durchschnittlich $61 \%$ der Fälle die maßgeblichen Belastungen identifiziert werden. Dies ist ein vergleichsweise hoher Wert, da in den meisten Fällen eine Überlagerung von Mehrfachbelastungen auftritt und aufgrund der additiven
Wirkungen bzw. Wechselwirkungen maßgebliche Belastungstypen sich grundsätzlich schwer erkennen lassen.

Ein ökologisch begründetes Kenntlichmachen der maßgeblichen Belastung ist sehr hilfreich, da die Identifizierung von Belastungen alleinig anhand von Belastungsinformationen nicht immer einfach ist. Bekanntlich ist die Verfügbarkeit qualitativ hochwertiger Belastungsdaten in Österreich noch immer sehr begrenzt. Zusätzlich ist daher zu gewährleisten, dass jene maßgeblichen Belastungen, die auch den ökologischen Zustand beeinträchtigen, erkannt und gezielt saniert werden, und nicht Geld in Restaurationsmaßnahmen investiert wird, welche nur einen bescheidenen Erfolg erwarten lassen.

Wesentliche Ergebnisse der Analyse der Belastungskombinationen sind im Regressionsbaum in Abbildung 4 dargestellt. Den größten Einfluss auf den fischökologischen Zustand besitzt die Landnutzung im Pufferstreifen $100 \mathrm{~m}$ links und $100 \mathrm{~m}$ rechts der Gewässer. Liegt der Anteil an Wald und extensivem Grünland im Pufferstreifen eines $10 \mathrm{~km}$ langen Flussabschnitts unter $53 \%$, so ist das Gewässer sehr stark beeinträchtigt. In diesem Fall folgt man dem linken Ast des Baumes. Innerhalb dieser sehr stark beeinträchtigen Probenstellen wird nun zwischen Gewässern mit mehr als $13 \%$ gestauten Bereichen innerhalb von $10 \mathrm{~km}$ und solchen, die weniger gestaute Bereiche aufweisen, unterschieden.

Liegt jedoch der Anteil an Wald und extensivem Grünland im Pufferstreifen über $53 \%$, so sind die Gewässer durchschnittlich in einem wesentlich besseren $\mathrm{Zu}$ stand, was die Mehrzahl der untersuchten Probestellen betrifft. In diesem Fall folgt man der rechten Seite des Baumes. Ist dann der ökomorphologische Zustand (Kriterium Sohle) des Flussabschnittes (5 km Länge) schlechter als 2,9, 
so schlägt sich dies auch in einem schlechten Fischindex von $-2,2$ nieder. Ist der ökomorphologische Zustand besser als 2,9, so hängt der fischökologische Zustand vom Grad der Gewässerfragmentierung und vom Staueinfluss ab. Gibt es in diesen Gewässern Abschnitte, die besonders lange Staue (Verhältnis Staulänge zu MQ >203) beinhalten, liegt der durchschnittliche Fischindex bei $-1,7$. Sind mehr als 10 Kontinuumsbarrieren pro Fischregion vorhanden, so liegt der Fischindex bei $-1,4$, sind es weniger als 10 so steigt er auf durchschnittlich $-0,8$. Der beste fischökologische Zustand wird daher in solchen Gewässerabschnitten erzielt, die einen hohen Anteil an Wald und Grünland, halbwegs gute ökomorphologische Bedingungen, keine oder nur wenige kleine Staue und weniger starke Fragmentierung aufweisen.

Das hier dargestellte Modell erklärt die Variabilität der Daten zu $42 \%$. Insgesamt sind 6 unterschiedliche Zustände bzw. Kombinationen von Belastungen beschrieben. Bis auf Restwasser sind alle behandelten Belastungen (Landnutzung, Regulierung, Stau und Kontinuum) im Modell enthalten. Die Distanz zwischen den Ebenen (vertikale Linien) sind ein Maß für den relativen Einfluss der Belastungsvariablen. Eindeutig erklärt die Landnutzung den höchsten Anteil am Gesamtmodell, gefolgt von Anteil gestauter Abschnitte sowie ökomorphologischer Zustand in $5 \mathrm{~km}$ langen Gewässerabschnitten. Verhältnis Staulänge zu MQ und Anzahl der Barrieren pro Fischregion spielen eine vergleichsweise untergeordnete Rolle.

Das Belastungskriterium Landnutzung integriert eine Reihe von Belastungen und ist wahrscheinlich deshalb für den gewässerökologischen Zustand so maßgeblich. Dazu zählen direkte und indirekte Wirkungen. Direkt wirksam wird z.B. die Beschaffung der Ufervegetation in Form von Beschattung,
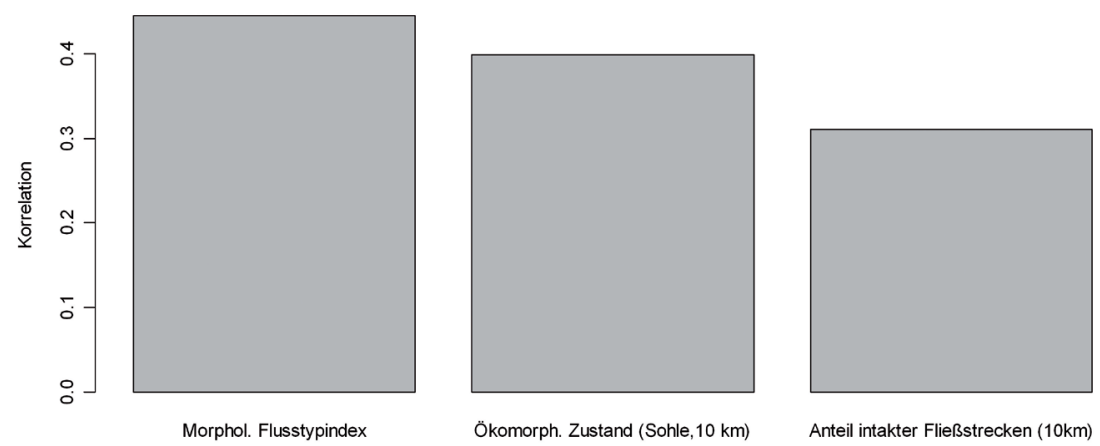

Abb. 5: Korrelationen ( $r>0,3)$ zwischen Landnutzung (Anteil Wald \& extensivem Grünland) im $100 \mathrm{~m}$ Puffer von $10 \mathrm{~km}$ langen Abschnitten und anderen Belastungskriterien

Sichtschutz, Fischeinständen (v.a bei Hochwasser), Totholz, etc. Indirekte Wirkungen sind v.a. durch die Pufferfunktion extensiv bewirtschafteter Uferrandstreifen gegeben: Reduktion des Eintrages von Nährstoffen sowie toxischer Stoffe aus angrenzenden Flächen, Abpufferung des Feinsedimenteintrages aus Ackerflächen, etc. Extensiv bewirtschaftete Pufferstreifen sind jedoch auch ein Indiz für höhere Überflutungshäufigkeit, was für die Fischfauna von Nutzen ist.

Weiters ist $\mathrm{zu}$ berücksichtigen, dass es einen deutlichen Zusammenhang zwischen Landnutzung und anderen Belastungen gibt (Abbildung 5). So zeichnen sich Gewässer mit extensiv bewirtschafteten Pufferstreifen meist durch vergleichsweise besseren ökomorphologischen Zustand aus und weisen insgesamt weniger anthropogene Einflüsse auf. So korreliert z.B. im zur Verfügung stehenden Datensatz die Qualität des Pufferstreifens am stärksten mit dem Index des morphologischen Flusstyps $(\mathrm{r}=0,45)$, aber auch mit dem ökomorphologischen Zustand (Gesamtindex auf $10 \mathrm{~km}: \mathrm{r}=0,40)$ und dem Kriterium Anteil ökomorphologischer $\mathrm{Zu}$ stand $>=2$ in $10 \mathrm{~km}$ langen Fließstrecken $(\mathrm{r}=0,31)$.

Dies zeigt, dass die Wahrscheinlichkeit des Vorliegens morphologisch intakter Zustände in Gewässern bei hochwertigen Pufferstreifen wesentlich größer ist als in Gewässern mit direkt angrenzender, intensiver Landwirtschaft und Siedlungen. Es gibt daher einen Anteil am Einfluss des Kriteriums Landnutzung, der direkt auf diesem Faktor beruht, und einen der indirekt auf Zusammenhänge mit anderen Belastungen zurückzuführen ist.

Die vergleichende Analyse der Einzelbelastungen zeigt, dass wiederum die Landnutzung den stärksten Modell-Erklärungswert hinsichtlich der Wirkung auf den ökologischen Zustand besitzt. Dies ist ein weiteres Indiz für die übergeordnete Bedeutung dieses Belastungstyps.

Ein Vorteil des Kriteriums Landnutzung ist dessen leichte und kostengünstige Erfassbarkeit. Mithilfe des österreichweit vorhandenen "Sinus-Datensatzes“ (Wrbka et al. 2003) ist eine grobe, flächendeckende Beurteilung der gewässerökologischen Situation vergleichsweise einfach möglich und sollte in Zukunft verstärkt umgesetzt werden. Insbesondere für die Übertragung von Detailergebnissen auf die österreichische Ebene anhand von Analogieschlüssen eignet sich dieses Kriterium sehr gut.

Wie bereits angedeutet, spielt neben der Landnutzung im Pufferstreifen die morphologische Veränderung infolge Regulierung eine wesentliche Rolle: Im GesamtModell ist der morphologische $\mathrm{Zu}$ stand als Zustand im $5 \mathrm{~km}$ langen 


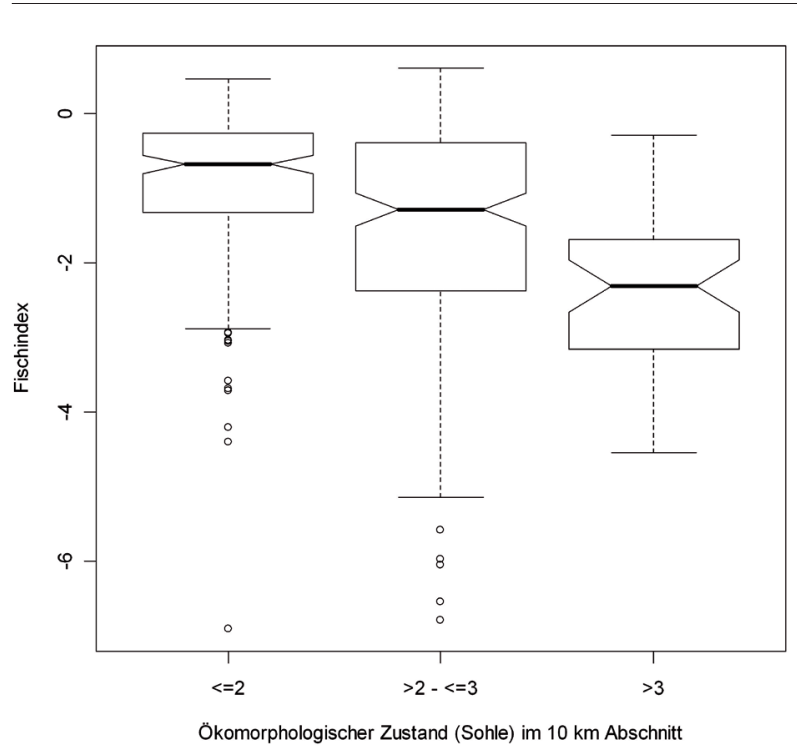

Abb. 6: Reaktion von Fischen auf den ökomorphologischen Zustand (NÖ-MORPH Kriterium Sohle [10 km]), nicht überlappende Einschnürungsbereiche stellen signifikante Unterschiede dar)
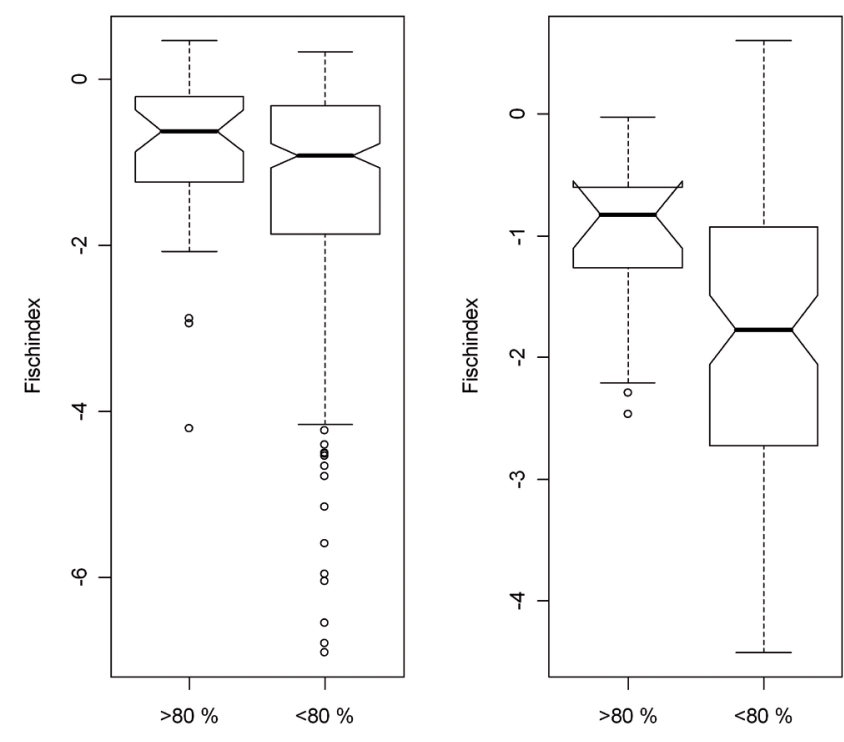

Abb. 7: Reaktion der Fische auf Prozent durchgängige Fließstrecken mit ökomorphologischem Zustand <=2 pro $10 \mathrm{~km}$ langem Abschnitt (links Rhithral, rechts Potamal)
Gewässerabschnitt vertreten. In der belastungsspezifischen Analyse tritt als stärkste Einflussgröße der Index des morphologischen Flusstyps hinzu. Dieses Kriterium schlägt aufgrund der hohen Redundanz mit der Landnutzung im Pufferstreifen, wie oben erklärt, erst bei der belastungsspezifischen Analyse durch. Ziel der morphologischen Sanierung muss daher sowohl eine zumindest teilweise Wiederherstellung des Flusstyps als auch ein nicht wesentlich veränderter ökomorphologischer Zustand sein. Als anzustrebenden Schwellenwert für den ökomorphologischen Zustand ist ein Index von 2 anzusetzen, da bereits ab diesem Wert Fische signifikant reagieren (Abbildung 6).

Sehr eindrücklich ist die Abhängigkeit vom räumlichen Maßstab erkennbar. Stärker beeinflussende Belastungen beziehen sich auf größere Maßstabsebenen (Einzugsgebiet und 5-10 km lange Abschnitte). Auch hierarchische Strukturen sind ersichtlich. Erst wenn auf größerer Maßstabsebene die Belastungen eine bestimmte Grenze unterschreiten, gewinnen lokale Belastungen an Bedeutung.

Für die räumliche Betrachtung kann das Belastungskriterium „Anteil an intakten Fließstrecken“, d.s. Fließstrecken mit einem ökomorphologischem Index $<=2$, herangezogen werden. In $10 \mathrm{~km}$ langen Gewässerabschnitten reagiert der Fischindex bereits bei einer Reduktion des Anteils intakter Fließstrecken unter $80 \%$ der Gesamtlänge (Abbildung 7).

Beim Belastungstyp Stau zeigen Fische eine Reaktion auf die Länge des Stauraums, absolut und in Relation zum MQ. Beide Kriterien spiegeln die Dimension des Staues wider. Kleine Staue ( $<300 \mathrm{~m}$ Länge) weisen einen besseren ökologischen Zustand auf als größere. Jedoch sind auch kleine Staue im Vergleich $\mathrm{zu}$ stauunbeeinflussten Situation als deutlich degradiert einzustufen. Hinsichtlich der Sanierung von Stauen bedeutet dies, dass v.a. die Dimension der Staue zu reduzieren wäre. Dies ist freilich in der Praxis nur in wenigen Fällen möglich.

Im Gesamt-Modell als auch im belastungsspezifischen Modell scheint das Kontinuum anhand von 2 Belastungskriterien auf: Anzahl der Kontinuumsunterbrechungen pro Fischregion und Prozent Fließ- strecken mit ökomorphologischem Zustand $<=2$. Beide spiegeln den Grad der Fragmentierung wider. Zugleich fällt bei beiden Kriterien die Reaktion der Fische im Potamal wesentlich stärker als im Rhithral aus. Auch anhand dieser Kriterien bestätigt sich, dass das Ziel der Sanierung die Schaffung möglichst langer, intakter und durchgehender Gewässerabschnitte sein soll. Relativ betrachtet, sollten Gewässerabschnitte zumindest über $80 \%$ intakte Fließstrecken aufweisen. In absoluten Zahlen sind deutliche Unterschiede zwischen durchgängigen Abschnittslängen von weniger und mehr als $4 \mathrm{~km}$ erkennbar. Dabei ist v.a. im Potamal auch bei längeren Gewässerabschnitten noch eine deutliche Beeinträchtigung gegeben. Daher sollten als Richtwert durchgängige Minimallängen von $5 \mathrm{~km}$ für kleine Rhithralgewässer und $10 \mathrm{~km}$ für restliche Gewässer angestrebt werden.

Obwohl die belastungsspezifischen Indizes klar zeigen, dass Fische auf Restwasser signifikant reagieren, scheint diese Art der Belastung im Gesamtmodell nicht auf. Dies kann mehrere Gründe haben. Erstens ist der zur Verfügung ste- 
hende Datensatz nicht ideal, um Gradienten zu untersuchen, da die überwiegende Zahl der Probestellen ähnlich geringe Restwasserführungen aufweist. Zweitens war die Beschaffung restwasserspezifischer Belastungsdaten (v.a. Restwasserabfluss) äußerst schwierig. Vielfach gründeten diese auf mündlichen Aussagen der Anlagenbetreiber und konnten im Rahmen des Projektes nicht vor Ort überprüft werden. Drittens belegen auch andere Restwasserstudien (EVN-Studie Zeiringer et al. in praep., Sabaton et al. 2004), dass es sehr schwierig ist, allgemein gültige Aussagen hinsichtlich Restwasserkriterien abzuleiten. Einschlägige Erfahrungen zeigen, dass auch viele andere Einflüsse, die im Rahmen dieses Projektes nicht erfasst wurden, die Situation in Restwasserstrecken bestimmen. So treten vergleichsweise häufig unterschiedliche Störungen beim Betrieb von Ausleitungskraftwerken auf (z.B. technische Gebrechen oder Fehlbedienungen der Dotationseinrichtungen), die zum kurzzeitigen Trockenfallen der Restwasserstrecken führen kann. Entsanderspülungen, kurzzeitige Absenkungen der Wehre und Stauraumspülungen sind weitere Belastungen, die die Fischpopulationen signifikant beeinträchtigen können.

Weiters ist $\mathrm{zu}$ berücksichtigen, dass die Auswirkungen von Restwasser sehr stark vom jeweiligen Gewässertyp abhängen. So zeigen jüngere Untersuchungen (z.B. Restwassermodellierung des IHG im Gesäuse), dass Fische in gefällsreichen Schluchtstrecken mit vergleichsweise wenig Restwasser das Auslangen finden.

Aufgrund dieser Situation wird daher vorgeschlagen, die Frage der Restwasserführung bzw. -dotation, wie bisher in Österreich üblich, fallspezifisch $\mathrm{zu}$ betrachten. Bei der Beurteilung der Restwasserfrage in den Entscheidungsbäumen des Instruments wird daher nur allgemein auf die Kriterien „Totalausleitungen“ und „ökologisch ausreichende Dotation“ eingegangen (Schmutz et al. 2007). Jedenfalls sollten keine Dotationen unter $40 \%$ MJNQT bewilligt werden, da hier Fische deutliche Reaktionen zeigen. Inwieweit im Einzelfall höhere Abflüsse erforderlich sind, kann anhand vorliegenden Datenmaterials nicht beurteilt und sollte daher fallbezogen bewertet werden

Wie die Ergebnisse zeigen, lässt sich anhand erfasster Belastungen nur ein gewisser Teil der Variabilität der Daten erklären. Dafür könnten z.B. andere Belastungen, die mangels Daten im MIRR Projekt nicht berücksichtigt wurden, wie z.B. der Fraßdruck durch fischfressende Vögel (Kormoran, Gänsesäger, Fischreiher), verantwortlich sein. Andere Faktoren können natürliche Schwankungen der Fischpopulationen, Unterschiede bei den Freilandbeprobungen, Einfluss der fischereilichen Bewirtschaftung, etc. sein.

\subsection{Entwicklung des Instruments}

Die Ergebnisse der Analysen liefern mehrere Bausteine, die sich nun zu einem strategischen Instrument für die Restauration von Fließgewässern zusammenfassen lassen (Abbildung 8). Als erster Baustein sind die belastungsspezifischen Fischindizes zu betrachten, anhand derer maßgebliche, d.h. ökologisch wirksame Belastungen identifiziert werden können. Dadurch wird erkenntlich, ob es sich um einfachoder mehrfachbelastete Gewässer handelt. Dies ermöglicht bei mehrfach belasteten Gewässern, wie sie in der Regel vorliegen, die Konzentration der Sanierungsmaßnahmen auf die maßgeblichen Belastungen. Die rein auf fischökologischen Daten basierende Identifizierung von Belastungen sollte durch verfügbare Belastungsdaten ergänzt bzw. überprüft werden.

Einen weiteren Baustein des
Instruments stellen die aus den Modellierungsergebnissen abgeleiteten und durch ExpertInnenwissen ergänzten Entscheidungsbäume für die Auswahl der Sanierungsmaßnahmen dar. Entscheidungsbäume und Schwellenwerte wurden für die Belastungstypen Regulierung (inkl. Landnutzung), Kontinuum, Stau sowie in genereller Form auch für Restwasser entwickelt.

Eine wesentliche Erkenntnis ist die additive Wirkung von Mehrfachbelastungen. Dies bedeutet, dass es zu einer Aufsummierung der Effekte der Einzelbelastungen kommt und daher mehrfachbelastete Gewässer durchschnittlich einen schlechteren ökologischen $\mathrm{Zu}$ stand aufweisen als Gewässer mit Einzelbelastungen. So reagieren Fische auf Kombinationsbelastung von Kontinuum und Regulierung um ein Drittel stärker als auf alleinige Belastung durch Kontinuumsunterbrechungen.

Für die Sanierung wiederum bedeutet dies, dass es in den meisten Fällen wahrscheinlich nicht ausreicht, nur die stärkste Belastung zu beseitigen, sondern grundsätzlich dieMehrfachbelastungzuentfernen ist (Sanierung aller Belastungen bis auf weniger wirksame). Dies führt jedoch meist zu wesentlich höheren Kosten. Im Sinne einer Priorisierung hinsichtlich Kosteneffektivität und zeitlicher Umsetzbarkeit wären daher Gewässer(abschnitte) mit Einzelbelastungen jenen mit Mehrfachbelastungen vorzuziehen. Wie schon oben festgestellt, betrifft dies jedoch nur einen vergleichsweise geringen Anteil der Gewässerstrecken, da die meisten mehrfachbelastet sind. Insgesamt betrachtet ist eine doppelgleisige Strategie am erfolgversprechendsten. Prioritär sind einfachbelastete $\mathrm{Ge}$ wässerstrecken $\mathrm{zu}$ sanieren, um vergleichsweise rasch und kostengünstig Sanierungserfolge vorweisen zu können; dies ist auch für die politische und öffentliche Akzeptanz von großer Bedeutung. 


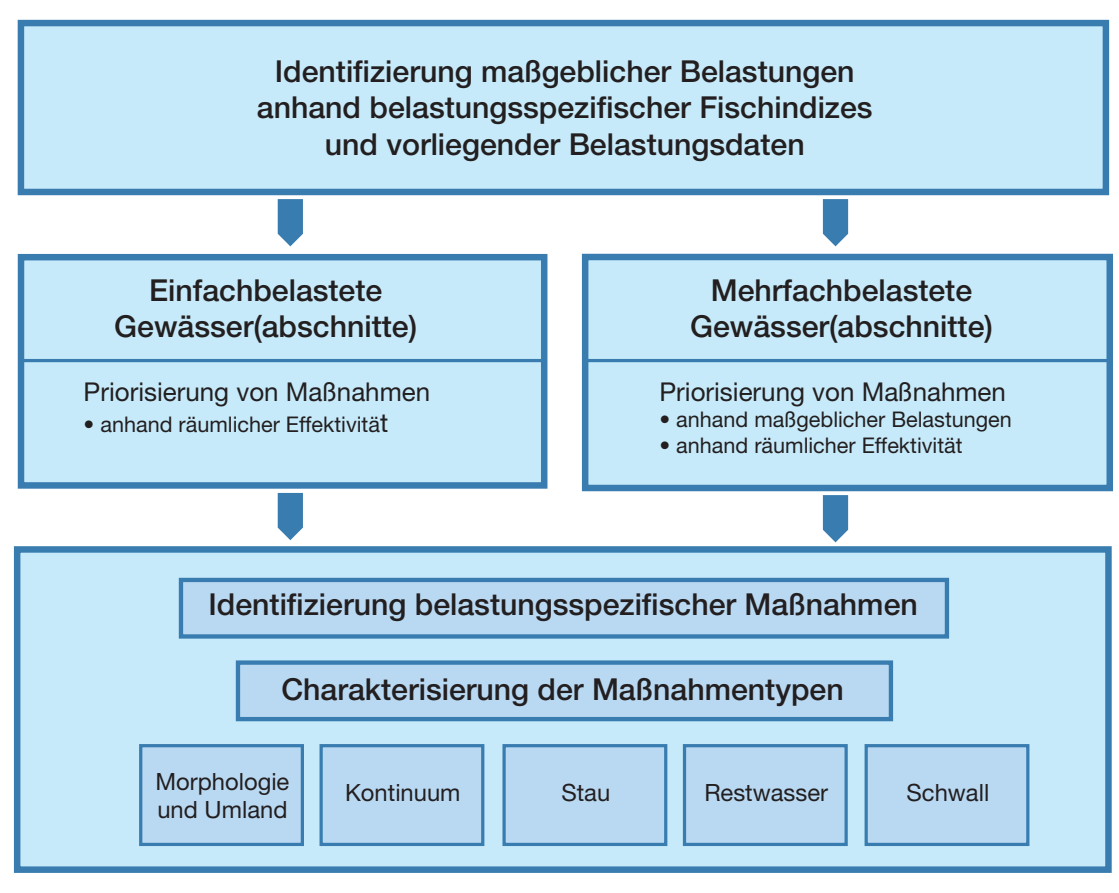

Abb. 8: Grundsätzliches Schema des Instruments zur Restauration von Fließgewässern

Parallel dazu ist die systematische Sanierung mehrfachbelasteter Gewässern anzugehen, um zumindest in absehbarer Zeit und mit dem Zeitplan der Wasserrahmenrichtlinie in Einklang stehend Sanierungserfolge vorweisen zu können.

\subsection{Priorisierung von Maßnahmen}

Aufgrund der Tatsache, das sehr viele Gewässer Österreichs ökologisch beeinträchtigt sind, ist es notwendig, die Sanierungen schrittweise anhand von Prioritätskriterien umzusetzen. Dabei ist entscheidend, wie effektiv die Maßnahmen im Bezug aufeinander sind (relative Effektivität) und welche räumliche Effektivität sie besitzen.

Im Falle der Einzelbelastung ist die Handlungsstrategie einfach, da direkt Maßnahmentypen, die der jeweiligen Einzelbelastung entsprechend entgegenwirken, angewendet werden können. Hier gilt es lediglich eine räumliche Priorisierung der Maßnahmen vorzunehmen.

Bei mehrfachbelasteten Gewässern hat auch eine Priorisierung hinsichtlich der Effektivität der belastungsspezifischen Maßnahmen zu erfolgen.

Wie die Ergebnisse zeigen, wirken sich die Belastungstypen sehr unterschiedlich auf den ökologischen Zustand aus. Im Sinne des dem Projekt zugrunde liegenden Analogieschlusses der Umkehrung des Degradationsprozesses in einen Restaurationsprozess bedeutet dies, dass auch die belastungsspezifischen Sanierungsmaßnahmen unterschiedlichen Einfluss auf den ökologischen Zustand besitzen.

Der ökologische Zustand der Gewässer ist maßgeblich durch Regulierung und intensive Landnutzung entlang der Gewässer beeinträchtigt. Dieser Belastungsfaktor ist beim zugrunde gelegten, jedoch nach Belastungstypen gewichteten Datenmaterial (Belastungstypen mit geringerer Häufigkeit wurden stärker gewichtet als häufigere) der am stärksten durchschlagende. Weitere maßgebliche Belastungen und Staue. Maßnahmen zur Sanierung von Stauen lassen sich nur beschränkt umsetzen. Es verbleisind Kontinuumsunterbrechungen ben somit die Morphologie, Landnutzung und das Kontinuum als wesentliche Systemkomponenten, für die sich allgemein gültige Aussagen ableiten lassen. Aufgrund der übergeordneten Bedeutung der Morphologie und der Umlandnutzung ist eine ausschließlich auf Sanierung von Kontinuumsunterbrechungen ausgelegte Strategie nicht zielführend, da sich dadurch der erwartete Erfolg hinsichtlich des ökologischen Zustandes nicht einstellt. Freilich gilt auch umgekehrt: erfolgt keine Sanierung der Kontinuumsunterbrechungen, ist das Ziel ausschließlich mit morphologischen Verbesserungen ebenfalls nicht erreichbar.

\subsection{Maßnahmenkatalog}

In einem Maßnahmenkatalog wurden geeignete Sanierungsmaßnahmen detailliert anhand einer allgemeinen Beschreibung sowie Beispielen dargestellt (Schmutz et al. 2007). Der Maßnahmenkatalog ist nach den Systemkomponenten Morphologie des Fluss-Auen-Systems, Hydrologie, Longitudinales Kontinuum und Einzugsgebiet gegliedert. Jede Systemkomponente ist in Maßnahmenkategorien, Maßnahmentypen und Maßnahmenbeispiele unterteilt.

\section{Ausblick und Empfehlungen}

Aus den Ergebnissen der Analysen lassen sich folgende allgemeine Empfehlungen ableiten:

- Entwicklung der Maßnahmenprogramme vom „Großen ins Kleine“, d.h. von der Ebene der Einzugsgebiete (Teileinzugsgebieten) hin zur lokalen Ebene.

- Priorität in der Sanierung sollten die weniger stark veränderten Einzugsgebiete erhalten, da dort die raschesten und größten Sanierungseffekte zu erwarten sind.

- Räumliche Priorisierung auf Potamalgewässer, da dort die 
Sanierungsmaßnahmen einen stärkeren Effekt zeigen.

- Maßnahmen mit großräumigen Effekten prioritär umsetzen (zum Beispiel Kontinuumsöffnung bei Mündungen)

- Bei mehrfach belasteten Gewässern - Sanierung aller maßgeblicher Belastungen (ausschließlich auf Sanierung von Kontinuumsunterbrechungen ausgelegte Strategie nicht zielführend)

- Ziel der Sanierung: möglichst lange, morphologisch intakte und durchgängige Gewässerabschnitte. Richtwerte für Mindestlängen bei kleinen Rhithralgewässern $5 \mathrm{~km}$, bei anderen Gewässern 10 km.
Die Ergebnisse wurden auf Basis von Daten des Bundeslandes Niederösterreich erarbeitet. Eine Übertragung der Resultate, sowie Anwendung des Restaurationsinstruments auf Gewässer des restlichen Bundesgebietes, ist nur für vergleichbare Gewässer hinsichtlich naturräumlicher Gegebenheiten und Belastungssituationen möglich. Für eine österreichweite Anwendung wäre die Liste der Gewässertypen vor allem um große alpine Fließgewässer zu erweitern. Zudem müssten Gewässer mit anderen Belastungen, wie z.B. Schwallbetrieb oder Stauraumspülungen, die im niederösterreichischen Datensatz nicht enthalten waren, ergänzt werden.
Schließlich sei an dieser Stelle auf das EU Projekt „EFI+, Improvement and spatial extension of the European Fish Index" (http:// efi-plus.boku.ac.at), einer Weiterführung des FAME Projektes (http://fame.boku.ac.at), verwiesen, welches sich mit der oben genannten Thematik in europäischen Fließgewässern auseinandersetzt.

\section{Korrespondenz:}

IHG - Institut für Hydrobiologie und Gewässermanagement; WAU - Department Wasser, Atmosphäre, Umwelt; BOKU - Universität für Bodenkultur, Wien

Max Emanuel-Strasse 17, 1180 Wien, Austria Phone: +43-1-47654-5223,

Fax: +43-1-47654-5217

http://mirr.boku.ac.at

andreas.melcher@boku.ac.at

\section{LITERATUR}

Haunschmid, R., Wolfram, G., Spindler, T., Honsig-Erlenburg, W., Wimmer, R., Jagsch, A., Kainz, E., Hehenwarter, K., Wagner, B., Konecny, R., Riedmüller, R., Ibel, G., Sasano, B. \& Schotzko, N. (2006): Erstellung einer fischbasierenden Typologie Österreichischer Fließgewässer sowie einer Bewertungsmethode des fischökologischen Zustandes gemäß EU-Wasserrahmenrichtlinie. Schriftenreihe des BAW Band 23,Wien.

Wrbka, T., Peterseil, J., Kiss, A., Schmitzberger, L Plutzar, C. Szerencsits, E, Thurner, B., Schnei-

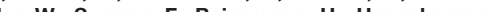
, W., Suppan, F., Beiss R. \& Tutsch, G. (2003): Landschattokologische Strukturmerkmale als Indikatoren der Nachhaltigkeit - Endbeicht zum Forschungsprojekt SINUS (Spatial lity). Wien. Forschungsprogramm „Kulturlandschaft“ - bm:bwk. 298.
Sabaton, C., Souchon, Y., Lascaux, J.M., Vandewalle, F., Baran, P., Baril, D., Capra, H., Gouraud V. Lauters, F., Lim, P., Merle, G. \& Paty, G. (2004): The "Guaranteed Flow Working Group": A French evaluation of microhabitat component of IFIM based on habitat and brown trout population monitoring. Hydroéco Appl. 14(1):245-270.

Schmutz, S., A. Melcher, S. Muhar, A. Zitek, M. Poppe, C. Trautwein, M. Jungwirth (2007): MIRR-Model C. Trautwed in. Jor River RestoratD. On. Entwicklung eines stramentschen Instruments z integrativen Bewertung okologischer Restaurations-

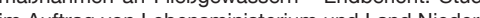
im Auftro von Lebensministerium - End Land Nederoac at/mirr resultate.
Zeiringer, B., Unfer, G. \& Jungwirth, M. (in praep): Durchführung von gewässerökologischen Untersuchungen im Bereich von Kleinkraftwerken der EVN Naturkraft.

Zitek, A. (2006): Potential criteria for modelling fish/ pressure relationship in running waters. A literature review. Studie im Auftrag von Lebensministerium und Land Niederösterreich, Wien. http://wasser.lebensmi-

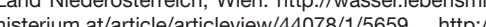
mirr.

Zitek, A. Haidvogl, G. Jungwirth, M., Pavlas, P. Zitek, A., Haidvogl, G., Jungwirth, M., Pavlas, P. \& Schmutz, S. (2007): Ein strategischer Leitfaden zu Wiederherstellung der Durchgängigkeit von Fließge wässern für die Fischfauna von Osterreich. Studie im Auftrag von Lebensministerium und Land Niederöster- 